\title{
Analysis of Embouchure and Breath Control of Bassoon
}

\author{
Xiaochuan Chang \\ Xi'an Conservatory of Music, Shanxi Xi'an, 710061
}

Keywords: Bassoon; embouchure; breath control

\begin{abstract}
Bassoon is an important instrument in the woodwind basso of western symphonies. Its unique structure, tone and shape provide the basis for the rich performance forms of western symphonies. During the process of bassoon performance training, mouth skill and breath control is an important basic skill training module. Therefore, this paper mainly focuses on the study of breath and embouchure during bassoon playing. Through the function and characteristics of embouchure and breath in bassoon playing, a simple analysis is made on the regulation of timbre and intonation in bassoon playing.
\end{abstract}

\section{Introduction}

Bassoon mainly originated in the 16th century. Bassoon tube is a woodwind instrument, which has the characteristics of wide range and mellow timbre. Through flexible playing skills, bassoon can show various forms such as long tone, tuning, vibrato and staccato. However, as the external shape and internal structure of bassoon are mainly composed of tube body and reeds, it has higher requirements on the basic skills, breath and embouchure application skills of the performers. Therefore, it is of great significance to properly analyze the basic embouchure and breath control skills in order to ensure a good presentation of mellow and bright timbre.

\section{Significance of research on embouchure and breath control of Bassoon playing}

In the study of the theoretical structure of bassoon, embouchure and breath are two independent modules, and they need to use different training methods. While in the specific performance process, only their good and close coordination can show the beautiful tone of bassoon. In the training process, bassoon skills are mainly taught by mouth and heart, and there is no systematic and scientific summary of theory, which has a negative impact on the training of bassoon learners, especially beginners. Therefore, in order to promote students to understand the correct embouchure and breath control methods, it is necessary to properly explore the embouchure and breath control during the performance of bassoon.

\section{Embouchure during Bassoon playing}

\subsection{Overview of the application of embouchure in the Bassoon playing}

Embouchure is the basis of good timbre performance, correct intonation and volume control for beginners of bassoon. In the process of bassoon playing, the embouchure is not discussed in specific words, which is mainly that the bassoon players use facial muscles and lips to display the range and intensity range under the control of nerve center ${ }^{[1]}$.

\subsection{Retention and application of correct embouchure in Bassoon playing}

During the Bassoon performance, the main embouchure is that upper and lower lip wrap the inner region, which is mainly in the upper and lower teeth tip. The face is in a natural state and provides support for the lip muscle movement. At the same time, the chin is slightly flattened, and the bassoon reed is included in the middle of the lips to control the overall product type relaxation and control the change of tone through the enhancement of lip movements. Bassoon instruments have three semi octave vocal range and only the fixed embouchure and facial muscle state can't 
guarantee the effective display of its vocal range. Generally speaking, during the basso performance, the bassoon player needs to control the lips to be in a relaxed state, minimize the pressure of the lips on the bassoon reed, and combine with the appropriate breath control to obtain the full bass intonation. In the treble stage, the lips should be as close as possible to the tip of the teeth, close to the gums. At the same time, the chin is slightly pulled in parallel state to increase the tension of the mouth and maintain the effect of lip support.

\subsection{Training method of correct embouchure of bassoon}

In the performance of long-piece music, if the correct embouchure cannot be mastered, it will lead to players' mouth muscle fatigue or lack of muscle maintenance. In order to avoid the above problems, on the one hand, in the actual training process, bassoon players can conduct long tone training at regular intervals every day, and consciously avoid such bad embouchures as bulging cheeks and biting reed. Because the physiological characteristics of bassoon players are quite different, the same embouchure may produce different performance effects. Therefore, during the practice of double lip wrapping, the viewer should be able to clearly see the lip wrapping reed.

On the other hand, in the specific performance stage, in order to ensure the accuracy of embouchure application, it is necessary to properly control the tension degree between lips and the reed, namely integrating of loose and tight. "Loose" mainly refers to keeping lips relaxed during playing the bassoon, avoiding putting extra pressure on the reed, or blindly exerting pressure on the reed, or biting the reed due to mouth fatigue. "Tight" is to maintain the sealing degree of the reed with embouchure, combined with sufficient breath control to avoid air leakage at the contact position between the reed and the lips and ensure the vibration effect of the reed. According to the material characteristics of bassoon reed, in the specific basic training process, players should maximize the vibration of the reed, and feel their own lips are always wrapping the reed on the basis of the lips completely wrapping the reed. Or they can open mouth to sing, and then control the vibration of the reed to determine the fusion node between singing and blowing.

In addition, in the process of embouchure training, attention should be paid to maintain the natural state of the whole face, that is, to control the face muscle to be in a relaxed state and the lip corner the most tense position while ensuring that the lips do not leak, so as to effectively improve the control function of the lip corner.

\section{Breath control in Bassoon playing}

\subsection{Characters of breath control in bassoon playing}

Breath control of bassoon is similar to respiration, which mainly refers to the physiological process of inhaling oxygen and discharging carbon dioxide, while breath is subconsciously controlling breathing rhythm. Through artistic respiration, different phrases and different emotions can be shown ${ }^{[2]}$.

\subsection{Methods of breath control in bassoon playing}

In the process of bassoon performance, the common breathing methods mainly include abdominal breathing, thoracic breathing and thoracic-abdominal breathing. Among them, thoracic breathing mainly uses the chest to control the breath. After inhaling from the lungs, the gas is discharged through external compression from the chest. During the application of thoracic breathing, obvious chest lifting, shoulder shrugging and other actions will occur when exhaling. Abdominal breathing mainly uses the diaphragm for breath control. That is to say, when inhaling, the waist and abdomen can be expanded to the maximum extent through the submergence of the diaphragm, and the air volume can be increased to the maximum extent by means of "breathing into belly". in the breath discharge stage of thoracic-abdominal breathing, the chest, waist abdomen, ribs will be expanded outward at the same time to ensure the maximum increase in inhalation amount of the following breath.

As the breathing habits of different players vary greatly, the learners need to ensure the 
maximum air capacity and the minimum muscle consumption according to their own performance characteristics in breath control training, that is, to reduce the body tension to the maximum extent, so as to guarantee the sufficient air capacity in the bassoon performance.

\subsection{Training of breath control skill in Bassoon playing}

In the stage of performance, the application of playing breath needs long and continuous repeated practice. Most beginners cannot keep long-time practice, leading to the failure of effective improvement in their performance skills ${ }^{[3]}$. In order to improve the full degree of tone of bassoon to the maximum extent, that is to say, the large volume contrast during the training of longer phrases. It is necessary to conduct the breath control training in actual practice.

The common breath control method of bassoon playing is to lie flat, relax the hands and place them on the abdomen, open the mouth at a slight angle to inhale and gradually feel the process of abdominal expansion. At the same time, hands lift up slightly under the influence of waist and abdomen tension, gradually looking for abdominal breath control nodes. In the training of abdominal breath control, attention should be paid to avoid the nose breathing, and subconsciously control the expansion of the lungs with the inhalation, and adopt even, slow exhalation and inhalation, gradually feeling the breath enter the abdomen. Otherwise, the body should be kept naturally upright and relaxed, with 2 legs in shoulder width. At the same time, both hands are put at the belly, and the mouth is slightly opened to inhale the breath into the abdomen. During this process, both hands can gradually feel the increase breath of the abdomen and determine the maximum air absorption in the abdomen. The breath is then gradually expelled from the mouth. Attention should be paid to control the expansion of the lung with the change of air volume in the inhalation stage, and avoid shrugging in the inhalation to ensure that the breath reaches the abdomen smoothly.

If the above methods cannot produce good results after basic training, Bassoon learners can bend over at $90^{\circ}$, put two hands at the abdomen, breathe in at an even and slow rate, and use hands to gradually feel the belly expansion. After the breath enters the abdomen smoothly, they can carry out the repeated practice, and return to stand upright, gradually looking for the control node when bending down and inhaling breath into the abdomen.

\section{Conclusion}

In conclusion, both the correct embouchure and the reasonable breath control should be guaranteed during the bassoon performance. The two are complimentary. If the embouchure is the string of the string instrument, then the breath is the bow. Beautiful music can be made through their friction and vibration. Therefore, according to the characteristics of bassoon oboe, learners can focus on the aspects of embouchure and breath during actual performance training, and lay a foundation for improvement of bassoon performance skills through regular correct embouchure maintenance and breathing exercises.

\section{References}

[1] Chen Tianyang. Analysis of embouchure skills of Bassoon performance[J]. Home Drama, 2016(9):88-88.

[2] Jia Hairui. Discussion on the importance of embouchure and breath control in Bassoon playing[J]. Musical instrument, 2016(6):54-55.

[3] Xu Qifeng. Brief talk on the basic skills and performance skills of Bassoon[J]. Northern Music, 2014(13):60-60. 